\title{
Signal detection and identification at successive stages of observation
}

\author{
JOHN A. SWETS, DAVID M. GREEN, DAVID J. GETTY, and JOEL B. SWETS \\ Bolt Beranek and Newman Inc, , Cambridge, Massachusetts 02138
}

\begin{abstract}
The relationship of signal identification to signal detection was examined in a series of experiments. The signals were idealized lines and patterns of lines in a spectrographic display. On each trial, progressively more of the complete spectrogram was exposed in successive observation intervals, and after each interval the observer made both detection and identification responses that were based on the accumulating evidence. One model we employed conceives of detection and identification as proceeding together over time as parts of a unified process. A second model used shows how the joint detection-and-identification ROC-a relative operating characteristic that relates the joint probability of correct detection and correct identification to the probability of a false detection-may be predicted from the simple detection ROC. Both models were supported by the data.
\end{abstract}

A series of experiments were conducted to examine the relationship of signal identification to signal detection at successive stages of observation of relatively long signals. The fundamental theoretical idea is that, with continued observation of each signal and/or noise pattern presented, detection and identification proceed simultaneously as parts of the same process. Moreover, the accuracy of detection performance and the accuracy of identification performance will grow together in a predictable way (Nolte, 1967). Specifically, at each stage of observation a relative (or receiver) operating characteristic (ROC) of a joint form that relates the probability of both a correct detection and a correct identification to the probability of a false detection can be predicted from the simple detection ROC, as a function of the number of possible signals. The main assumptions of the underlying model are that the signals are orthogonal and of equal energy (Starr, Metz, Lusted, \& Goodenough, 1975). In general, the use of the ROC provides a way of examining the results of simultaneous detection and identification tasks that is independent of the observer's criterion for a detection response (Lindner, 1968).

The signals used in the experiments reported here met the criteria of independence and equal energy. They were highly idealized visual representations of underwater sounds, suggested by the spectrographic display used in some sonar applications. Two companion studies, reported separately, used more com-

This research was supported by a contract with the Engineering Psychology Programs, Office of Naval Research. We thank Elizabeth Graham, Lynda Santoro, and Jessie Kurzon for their dedicated service as observers in the several experiments, and Barbara Freeman for her able programming assistance. plex and correlated signals, in order to simulate more closely real (including underwater) sounds of practical interest. In the latter studies, an attempt was made to relate identification and detection performances by means of a multidimensional scaling analysis, and by means of developing correspondences of psychological and physical dimensions (Getty, Swets, Swets, \& Green, Note 1; Swets, Green, Getty, \& Swets, Note 2).

\section{THEORY}

In this paper, we use Nolte's (1967) theory of the "adaptive optimum receiver" in a heuristic, nonquantitative way, and this theory is simply characterized here at an appropriate conversational level. On the other hand, we use the theorem of Starr et al. (1975) to make quantitative predictions of our experimental results, and their equations are reproduced in this section.

Nolte suggested that the "adaptive optimum receiver" stores updated probability estimates separately for each signal under consideration, so that detection and identification proceed together in a unitary process that is the basis for responses of either kind. According to this conception, detection does not precede identification, or vice versa. There is no partial "aha" effect in either direction: the observer does not say, "Now that I know a signal is present, I can begin to determine which one," or, "Now that I know which signal I am observing, I can begin to build up detectability."

Broadbent (1971) has provided the analogy of an array of test tubes, each corresponding to a signal alternative, and each partly full of water. The selection of one tube corresponds to perception of a signal, 
and the probability of selecting a tube depends on how full it is. The initial level of water in each tube represents the bias toward recognizing the corresponding signal, and the presentation of a signal causes the level of the water in its tube to rise, to an extent depending on the strength of the signal and the sensitivity of the receiver.

In our first experiment, each of eight possible signals was a single vertical line, corresponding to energy in one of eight different frequency bands; i.e., a line in any one of eight different locations could occur on a given trial. In our second experiment, each of five possible signals consisted of 3 lines; i.e., any one of five sets of 3 different, particular lines (making 15 different lines in all) could occur on a given trial. In terms, then, of Broadbent's description of our model, we can imagine the receiver to utilize eight tubes when confronted with the eight signals of Experiment 1. In Experiment 2, to extend this conception, the observer might supplement the (five) "signal" tubes with an array of (15) "dimension" or "feature" tubes, with water levels representing the energy in different locations (or frequency bands). He might then pour the dimension tubes (with replacement) according to the predetermined patterns into the signal tubes.

We note that Nolte's and Broadbent's models apply to the true sequential decision task in its complex and realistic form, that is, where the observer decides when to declare whether or not a signal exists. The sequential observer determines, in effect, how full any tube must be to be selected, or how empty they must all be to indicate that no signal is presentperhaps easing both criteria as time passes. Our present experiments bypassed such a speed-accuracy tradeoff, by using trials of fixed length, so that we could focus better on the process of accumulating sensory information. As far as human detection is concerned, treatments of the sequential, or deferreddecision, task have appeared elsewhere (e.g., Swets \& Birdsall, 1967).

Nolte's and Broadbent's models also permit the various signals to have different prior probabilities and different utilities, and our present experiments avoided these complications too. We would point out, however, that these decision-theory models permit treating another realistic task-one in which responses are solicited from the observer at various times during observation, times that are determined by considerations possibly outside of the viewing environment. The varying prior probabilities and utilities may be supplied to the observer at the time that the response is solicited.

As suggested earlier, we have not devised a strong test of Nolte's quantitative theory, or of alternative theories. We advance the theory here as a general framework that may be useful in thinking about detection and identification in a unified way; it gives promise of treating identification in terms that have been successful in explicating detection. For now, we shall regard data showing detection accuracy and identification accuracy to grow smoothly together over time, as consistent with the framework. We shall consider a result that information is conveyed by second choices in identification to be another datum supporting the general conception: such a result would indicate that the observer collects and updates probability data on several signal possibilities, and has access at any time to more than the largest probability estimates. One substantive question we shall ask in this connection is whether or not the process of extracting, codifying, storing, and retrieving sensory information is improved by a particular aid to memory, namely, continued visual access to all of the stimulus information presented throughout the successive observation stages of a given trial.

Starr et al. (1975) have also drawn on modern detection theory to develop their model, one that we apply quantitatively in our attempt to relate identification and detection at successive stages of observation. They proposed and supported the model for visual localization, or the identification of a visual signal's location, and we are interested in exploring possible extensions of the model to forms of identification or classification not purely, or not so obviously, locational. Though the task of our Experiment 2 (with signals consisting of different sets of three lines) can be considered one of localization, such signals simulate spectrographic patterns and are hence suggestive of more general identification tasksincluding, for example, visible speech. Indeed, a reasonable extension of the present line of research would test the model with data on the audible correlates of our spectrographic signals. The general importance of the development by Starr and colleagues, as we see it, is that it is the first apparently tractable means of extending the ROC concept in detection theory to treat tasks involving multiple signal alternatives. Early attempts to do so (e.g., by Swets \& Birdsall, 1956) achieved rather limited success.

The theorem of Starr et al., shows how the probability of a "hit" or true-positive response, corresponding to each possible probability of a "false alarm" or false-positive response, is decreased when the "hit" response is redefined to include correct identification as well as correct detection. The extent of the predicted decrement depends on the number of possible signals; as mentioned, the model assumes that the signals are orthogonal and of equal energy. The so-called "simple" detection ROC that serves as the basis for predicting the "joint" ROC is the detection ROC that is obtained under uncertainty about which of the possible signals will be presented-not the ROC that is obtained for a signal known exactly. The relationship between the two kinds of detection ROC is discussed by Nolte and 


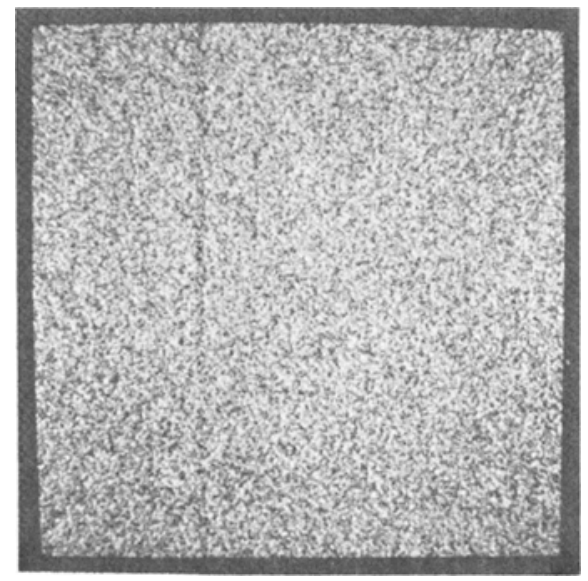

Figure 1. Experiment 1. Enhanced display of signal No. 3: a line in Position 3.

Jaarsma (1967) and by Swets and Birdsall (1978).

Starr and colleagues showed that the false-alarm probability is unaffected in moving from the detection ROC to the joint ROC:

$$
\mathrm{P}_{\mathrm{DI}}\left(\mathrm{S} \mid \mathrm{n} ; \mathrm{x}_{\mathrm{c}}\right)=\mathrm{P}_{\mathrm{D}}\left(\mathrm{S} \mid \mathrm{n} ; \mathrm{x}_{\mathrm{c}}\right) \text {, }
$$

where D indicates "detection only," DI indicates "detection and identification," $\mathrm{S}$ denotes a "signal present" response, $n$ denotes a "noise only" presentation, and $x_{c}$ denotes the variable decision criterion. The correct, joint "hit" response (S, CI), when a signal (s) is presented, can be shown to have a probability

$$
\begin{aligned}
P_{D I}\left(S, C I \mid s ; X_{c}\right)= & P_{D}\left(S \mid s ; x_{c}\right)-\left[\frac{M-1}{M}\right] \int_{0}^{P_{D}\left(S \mid n ; x_{c}\right)} \\
& \times\left[\frac{1-P_{D}(S \mid s ; t)}{1-P_{D}(S \mid n ; t)}\right] d P_{D}(S \mid n ; t),
\end{aligned}
$$

where $M$ denotes the number of possible signals, and $t$ is a dummy variable representing the various decision criteria that yield the simple detection ROC. An appendix to the article by Starr et al. (1975) may be consulted for the derivation of these equations.

Table 1

Signal Line Patterns (Experiment 2)

\begin{tabular}{cccc}
\hline & \multicolumn{3}{c}{ Location } \\
\cline { 2 - 4 } Signal & Line 1 & Line 2 & Line 3 \\
\hline 1 & 5 & 9 & 12 \\
2 & 2 & 7 & 10 \\
3 & 4 & 6 & 15 \\
4 & 3 & 8 & 16 \\
5 & 1 & 11 & 14 \\
\hline
\end{tabular}

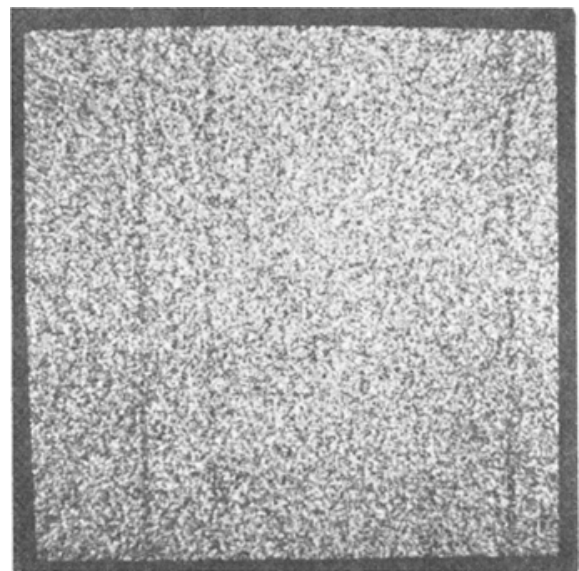

Figure 2. Experiment 2. Enhanced display of signal No. 3: a pattern with lines in Positions 4, 6, and 15.

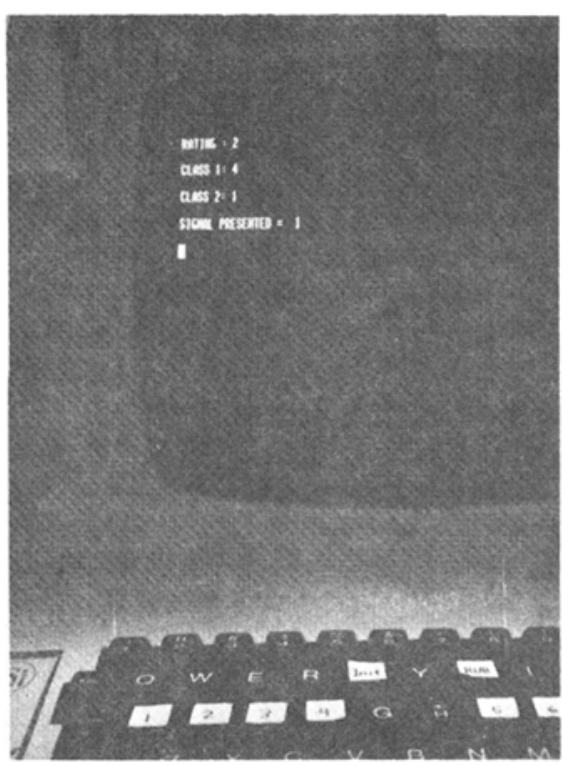

Figure 3. An example of the complete terminal display at the end of a trial in both experiments.

\section{EXPERIMENTS}

\section{Method}

\section{Description of the Signals and the Noise}

The signals and noise were generated on a DEC PDP-11/34 minicomputer driving a COMTAL 8000-SA image-processing and display system. The COMTAL generates an image consisting of $512 \times 512$ picture elements (pixels), in which each pixel can take on any of the 256 gray levels between black and white. The raster-scanned image is displayed in an area $24 \times 24 \mathrm{~cm}$ on a CONRAC 17-in. (43-cm) SNA television monitor.

The noise background consisted of a $256 \times 256$ element matrix, each noise element being a $2 \times 2$ square of pixels. Each element was assigned a gray value drawn randomly from a Gaussian distribution with a mean of 128 units on the COMTAL gray scale and a standard deviation of 25 units. The contrast and brightness controls on the CONRAC monitor had been adjusted such that the middle gray (128 units) corresponded to a luminance of about 
(a) WITH VISIBLE MEMORY NORMAL DEVIATE
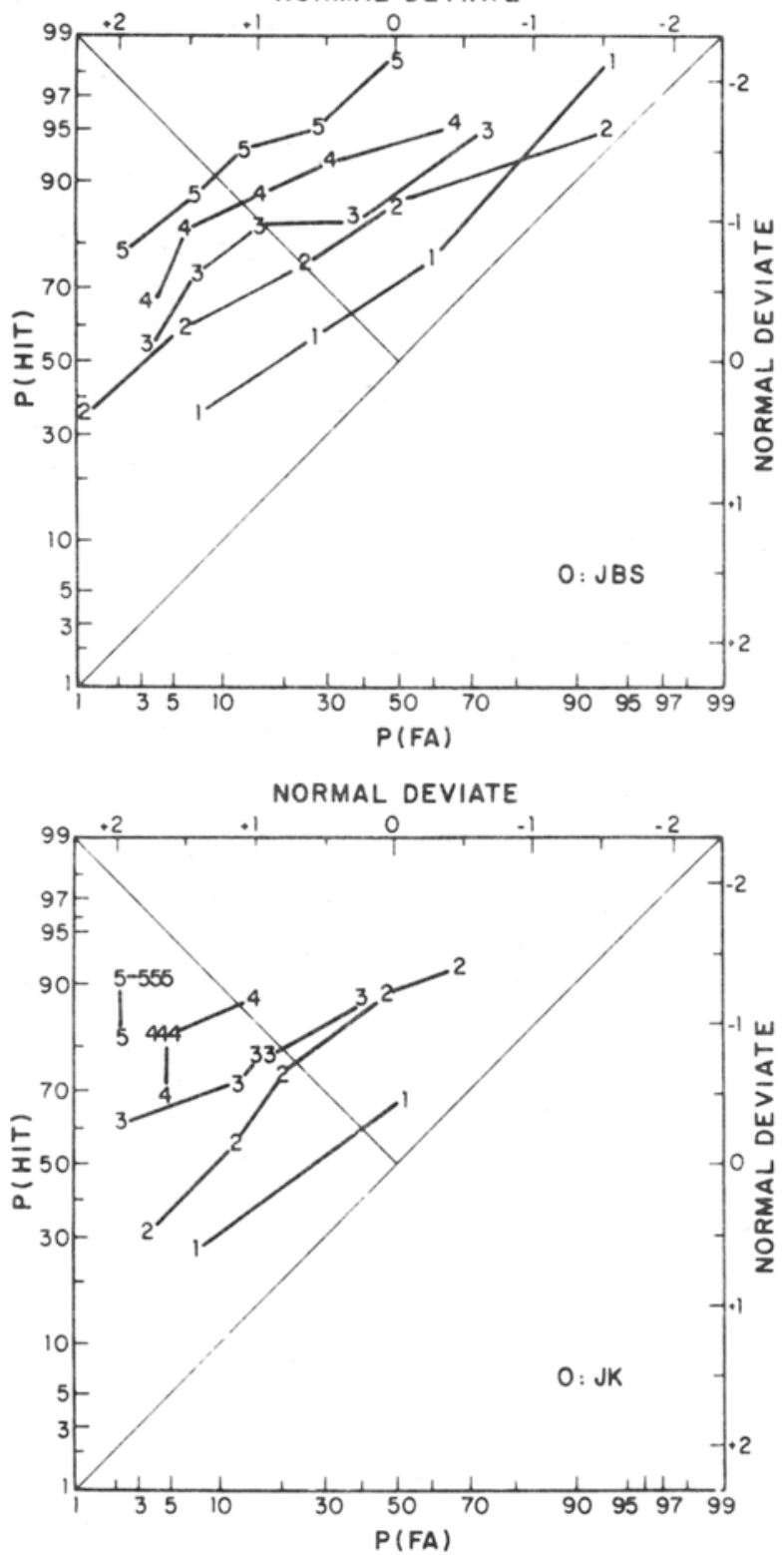

(b) WITHOUT VISIBLE MEMORY NORMAL DEVIATE
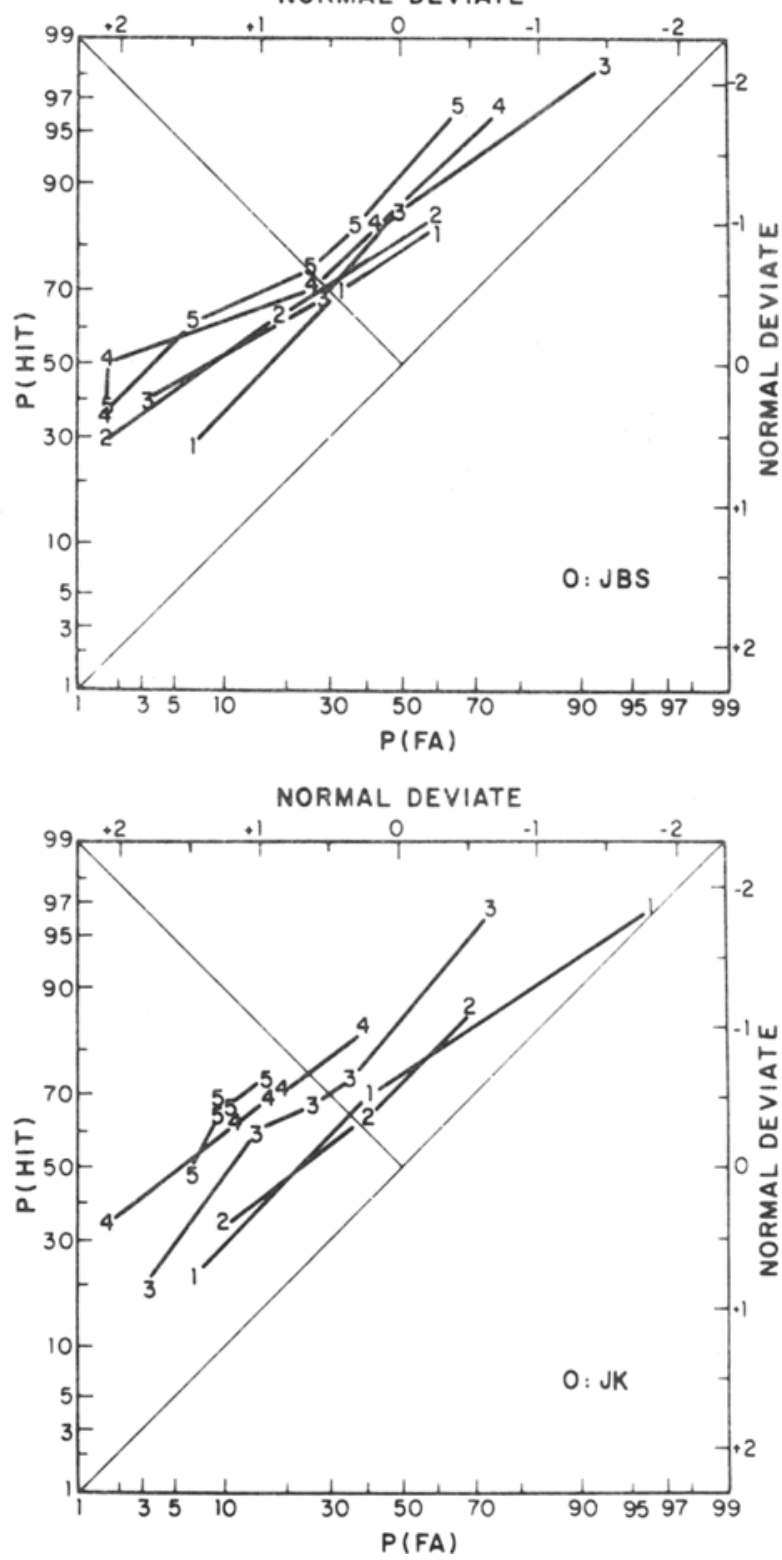

Figure 4. Pilot experiment. Detection ROCs for each of the five stages of observation for two observers: (a) with and (b) without visible memory.

$62 \mathrm{~cd} / \mathrm{m}^{2}$ and full white (255 units) corresponded to a luminance of about $308 \mathrm{~cd} / \mathrm{m}^{2}$.

The noise background was sampled anew on each trial. Signals, when present, were superimposed on the noise background by constructing a matrix of signal values and then displaying the sum of the signal and noise matrices.

In Experiment 1, eight signals consisted of single, vertical lines eight pixels in width, which differed in horizontal location across the display. The signal lines darkened the underlying noise background by five gray units ( $1 / 5$ of the noise standard deviation), and were centered horizontally in successive eighths of the display.
The general location of each of the potential signal lines was indicated to the observers by a horizontal strip above the image labeled with the digits 1 through 8 above successive signal-line locations. As an illustration, a slightly enhanced display of Signal 3 (a line in Position 3 ) is shown in Figure 1.

In Experiment 2, the display was divided horizontally into 16 locations. A set of five orthogonal signals was constructed by choosing five sets of three lines from the set of 16 , without replacement. The pattern for each of the five signals is given in Table 1, and an enhanced display of one of them (Signal 3) is shown in Figure 2. Each line was 8 pixels in width and darkened 

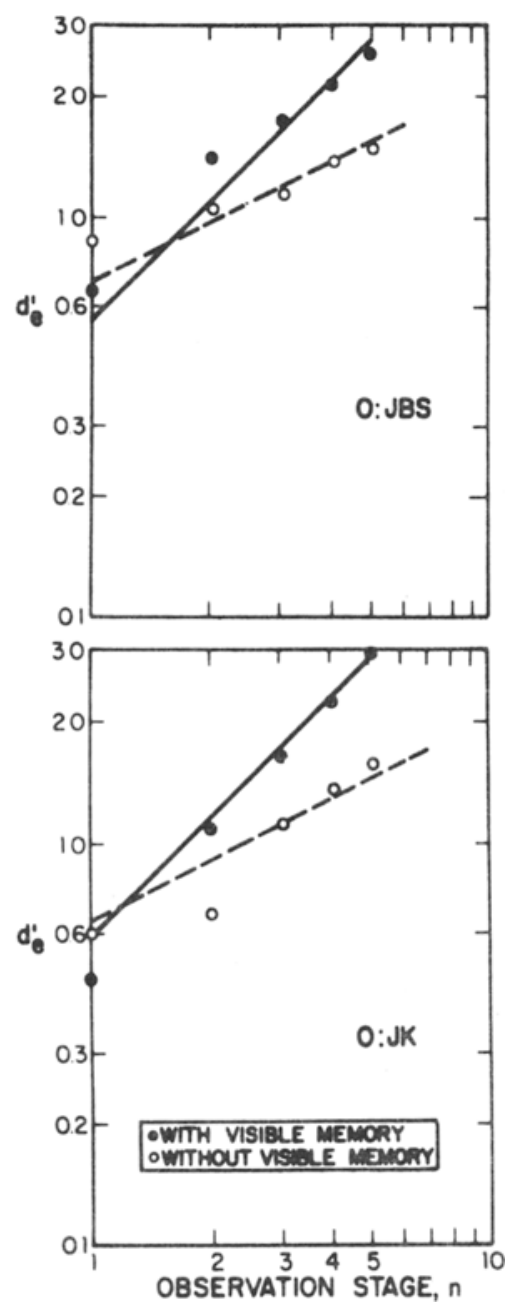

Figure 5. Pilot experiment. The detection index, $d_{e}^{\prime}$, over the five observation stages for two observers, both with and without visible memory. Best-fitting (least-squares) lines with slopes of one and of one-balf are shown as references for the visible and no-visible memory conditions, respectively.

the underlying noise by 3 gray units (less than $1 / 8$ of the noise standard deviation).

\section{Viewing Environment}

Observers sat approximately $2 \mathrm{~m}$ from the stimulus-display screen. This screen was about $1 \mathrm{~m}$ from the floor, and was viewed comfortably over the CRT/keyboard computer terminals (Lear Siegler ADM-3A) used for response cueing and response entry. Ambient room lighting was approximately $3 \mathrm{~cd} / \mathrm{m}^{2}$.

\section{Stimulus Presentation}

Signals were presented in noise at random on one-half of the trials, and noise alone was presented on the remaining trials. When a signal was presented, it was equally likely to be any one of the signals used in the experiment.

Each trial contained five stages of observation, with each stage followed by the responses described below. A stage consisted of painting a horizontal stripe over approximately the top one-fifth of the screen. Stages followed from top to bottom of the screen in "waterfall" fashion, each stage "pushing down" the preceding stages.

\section{Responses}

The first response made at each stage was a detection response in the form of a six-category rating of confidence. Following this response were first and second choices relative to identification, which were made no matter which detection response was made previously. Responses were made via the keyboard of the CRT terminal, with appropriate type and time of response cued by the terminal's display; the complete terminal display is shown in Figure 3.

\section{Trial and Session Timing}

A fifth of the screen was painted in $10 \mathrm{sec}$. The next fifth was painted after all observers had completed their responses. The observer-terminated response interval lasted approximately $5 \mathrm{sec}$, followed by a warning sound that the next stage would occur. Feedback was given at the conclusion of a trial, and $1.5 \mathrm{sec}$ intervened between trials.

Ten trials were presented in a block, and, as a rule, six blocks were presented in a $2-\mathrm{h}$ session. Thirty sessions were conducted over 8 weeks. Certain sessions or initial parts of sessions were designated as practice and not included in the analyses.

\section{Experimental Control}

Stimulus presentation and trial timing were controlled by the PDP-11/34 computer, which also recorded responses and analyzed the data.

\section{Observers}

The three observers were two high school students and one of the experimenters (J.B.S.).

\section{Experimental Conditions}

In Experiment 1, with eight signals consisting of single lines differing in location, two conditions examined the effect of (a) leaving visible and (b) erasing each of the five stages of stimulus presentation as these stages proceeded on a trial. A pilot study, employing J.B.S. and another technical assistant as observers, was essentially identical to Experiment 1. In Experiment 2, with five signals consisting of variously distributed three-line patterns, only Condition a was conducted.

\section{Pilot Experiment}

\section{Results}

Detection ROCs. Detection ROCs for each of five stages-(a) with and (b) without "visible memory" -are shown for the two observers on doubleprobability scales in Figure 4 . The two conditions were based on (a) 142 trials and (b) 120 trials. The form of the several ROCs-approximating a straight line with a slope perhaps less than but near unityappears reasonable, given the relatively small number of trials.

Detection accuracy over time. To ascertain how detection accuracy grew over time, we estimated the detection index $d_{e}^{\prime}$ visually from the graphs of Figure 4; $\mathrm{d}_{\mathrm{e}}^{\prime}$ is the normal-deviate index taken at the negative diagonal (as described by Green \& Swets, $1966,1974)$. The values of this index over the five stages are shown on double-logarithmic scales in Figure 5. As a reference for the visible-memory condition (a), the best fitting (least-squares) line with a slope of one has been drawn through the data points, representing a growth of $\mathrm{d}_{\mathrm{e}}$ proportional to the number of preceding stages, $n$. As a reference for 
(a) WITH VISIBLE MEMORY
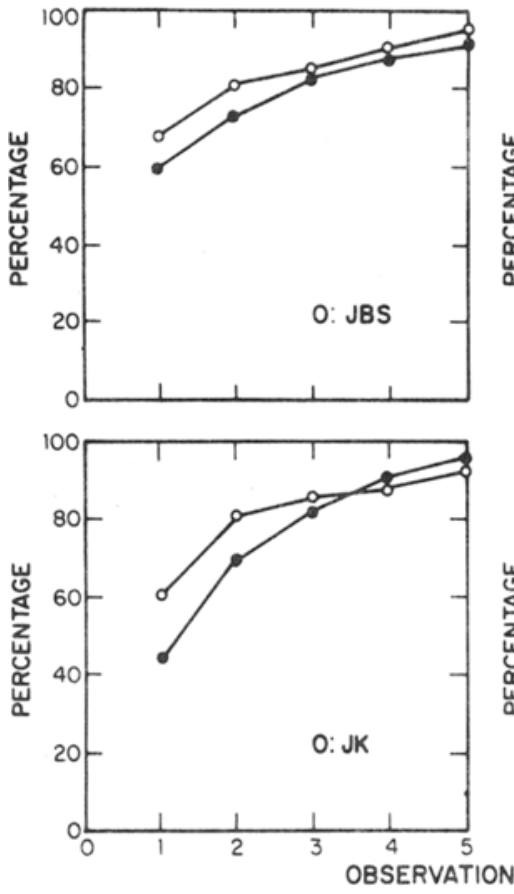

(b) WITHOUT VISIBLE MEMORY
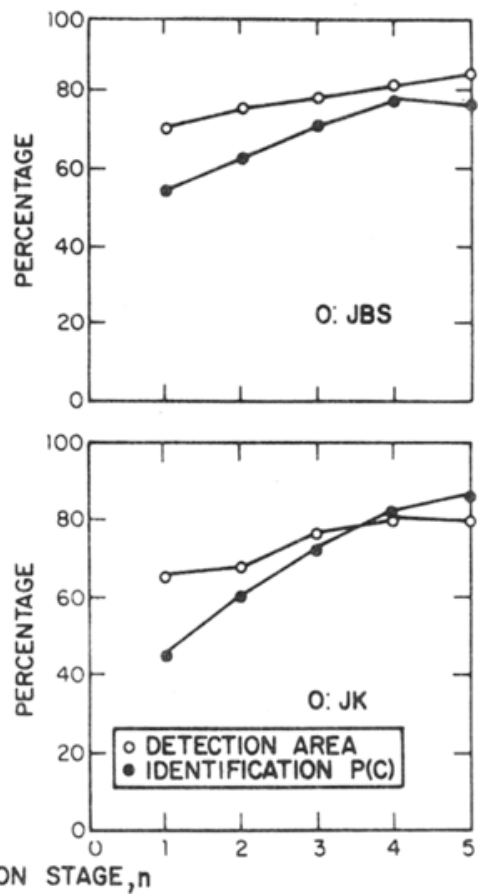

Figure 6. Pilot experiment. The area under the ROC curve (detection) and the percentage of correct responses (identification) over observation stages for two observers: (a) with and (b) without visible memory.

Condition $b$, the best fitting line with a slope of onehalf has been drawn through the data points, representing growth in $\mathrm{d}_{\mathrm{e}}^{\prime}$ proportional to the square root of $n$. Though we do not pursue the matter further here, we mention that the former prediction is consistent with some other results of areal summation in vision, and the latter prediction is consistent with data of several previous experiments in which integration depended on the observer's memory (Green \& Swets, 1966, 1974, chapter 9).

Detection and identification accuracy over time. Figure 6 shows detection accuracy-here indexed by the trapezoidal area under the ROC-over time, along with identification accuracy-here indexed by the percentage of correct responses-over time. The indication here is that the two processes proceed simultaneously, spanning together the range from a little more than chance to near perfect performance.

Predicting identification from detection. Joint detection-and-identification ROCs, having as ordinate value the probability of responses correct with regard both to detection and identification, are shown in Figure 7 . The figure shows the values predicted from the data points of the simple detection ROC, and the values obtained, at each stage of observation. There is good agreement between the two sets of values.

\section{Experiment 1}

Detection ROCs. Detection ROCs for each of five stages, under the two memory conditions, are shown for the three observers in Figure 8 . The two conditions were based on (a) 126 trials and (b) 141 trials. The more experienced observer yielded tidy data. The new observers yielded fairly regular ROCs for the condition with visible memory; without visible memory, the effective signal strength for them was quite low.

Detection accuracy over time. Figure 9 shows a growth of $\mathrm{d}_{\mathrm{e}}^{\prime}$ with visible memory having a slope somewhat less than unity. The data based on no visible memory have a slope only slightly less than those obtained with visible memory, and noticeably greater than one-half. Swets and Birdsall (1978) point out that signal uncertainty may produce an effect seen here in both sets of data but more clearly in the data based on no visible memory: a slope greater than one-half for early observations, during a phase of zeroing in on the location of the signal, and a slope near one-half on later observations, when location uncertainty is lessened.

Detection and identification accuracy over time. Detection area and identification percent-correct are shown for the two memory conditions in Figure 10. They proceed apace over a substantial part of the range from chance $(50 \%$ and $121 / 2 \%$, respectively) to perfect performance $(100 \%)$.

Accuracy of second choices. The percentages of correct second choices relative to identification, when 
(a) WITH VISIBLE MEMORY

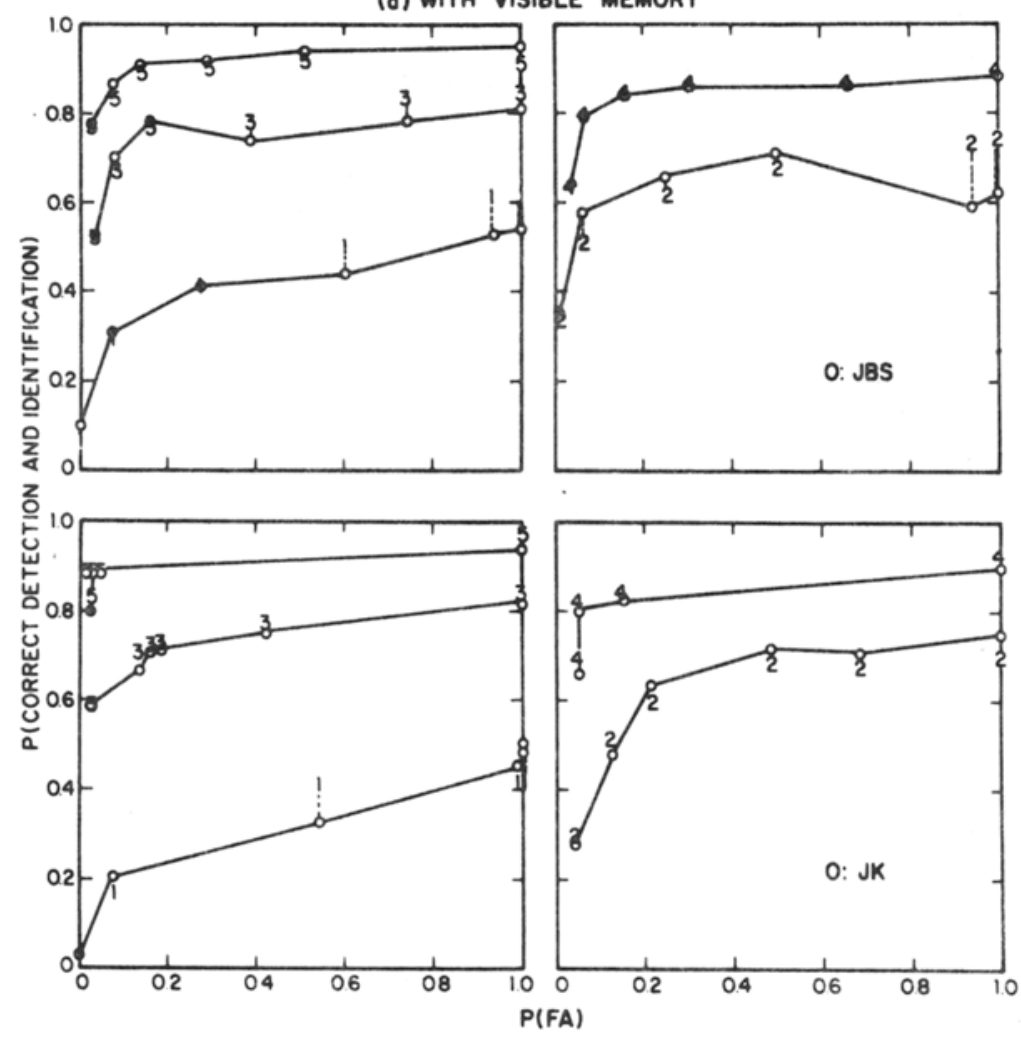

(b) WITHOUT VISIBLE MEMORY

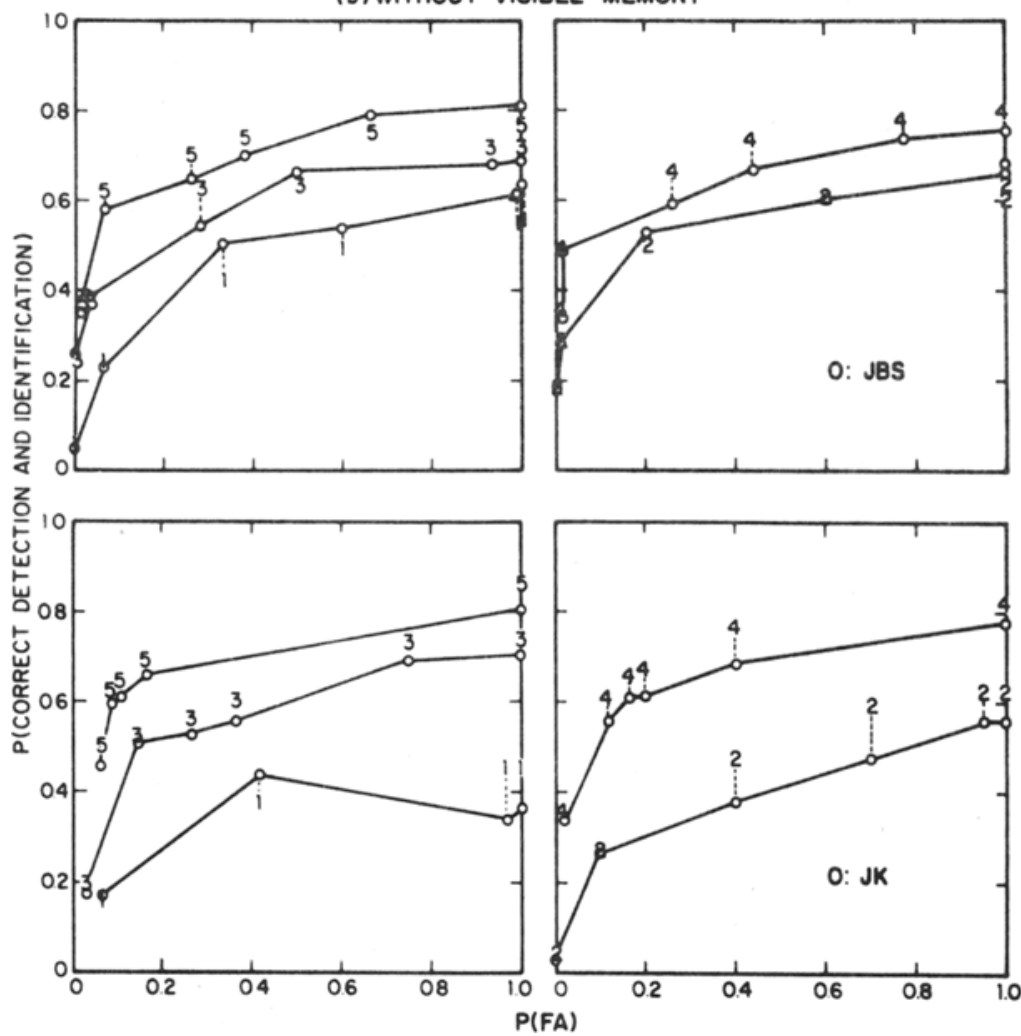

Figure 7. Pilot experiment. Joint detection-and-identification ROC curves for two observers for each of the five observation stages: (a) with and (b) without visible memory. Odd and even observation stages are presented in separate panels for the sake of clarity. The connected circles show values predicted by the model. 
(a) WITH VISIBLE MEMORY NORMAL DEVIATE
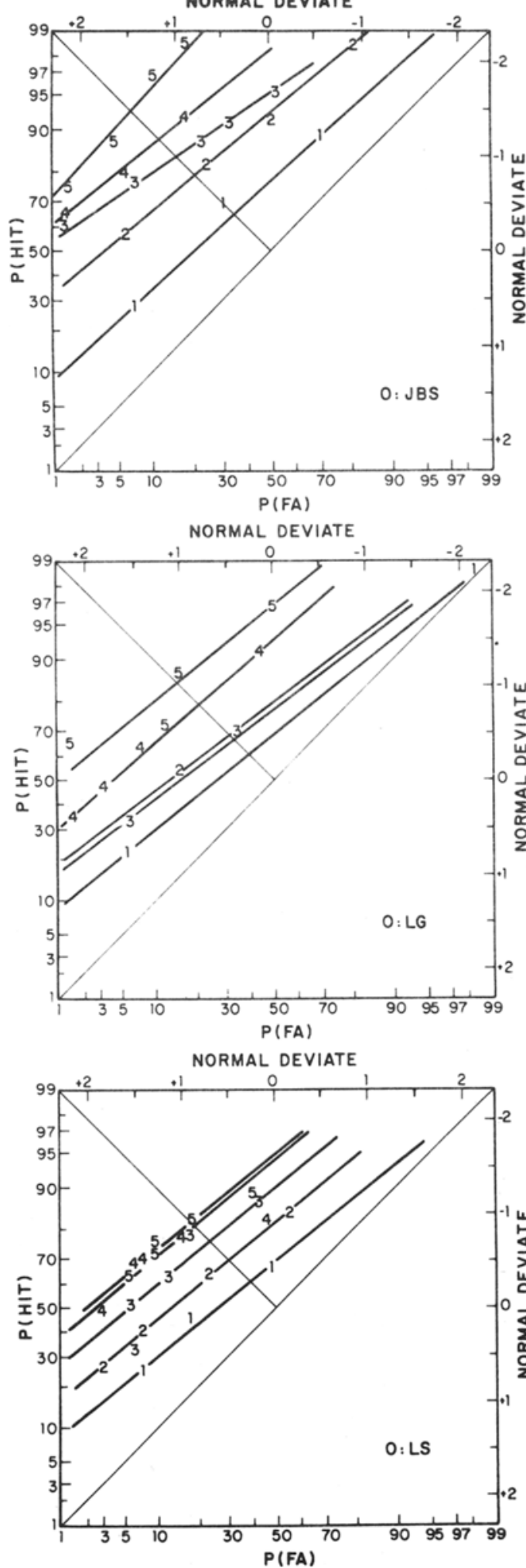

(b) WITHOUT VISIBLE MEMORY
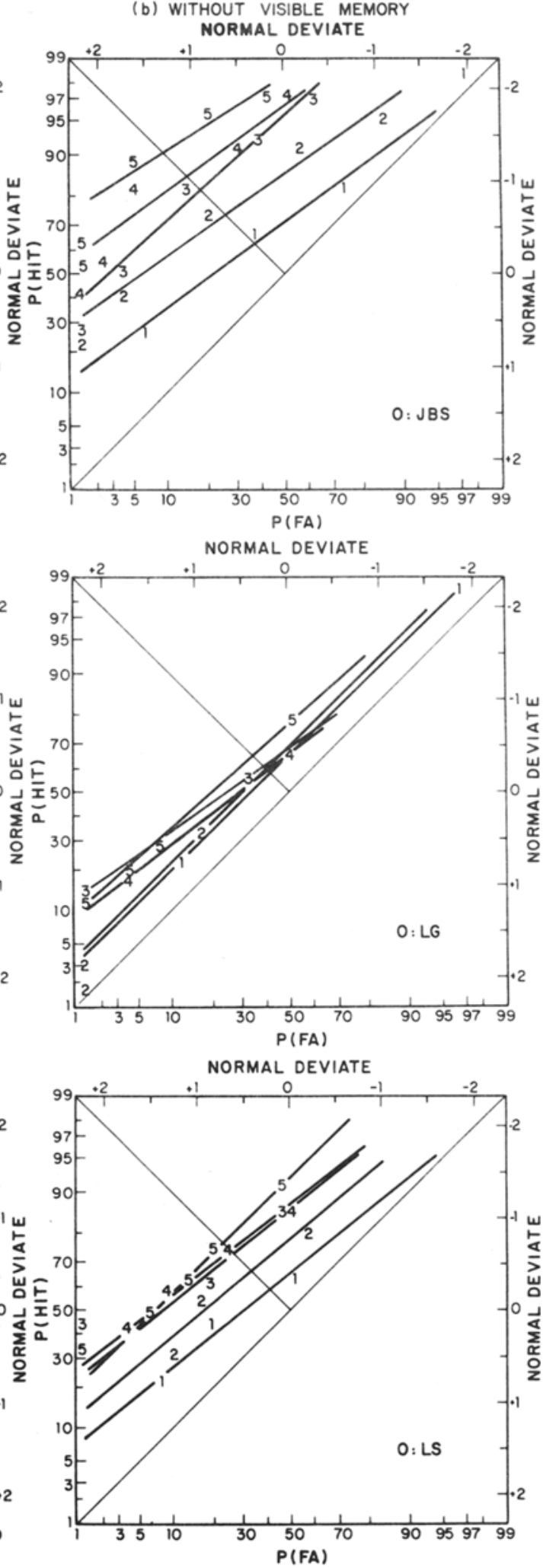

Figure 8. Experiment 1. Detection ROCs for each of the five stages of observation for three observers: (a) with and (b) without visible memory. 

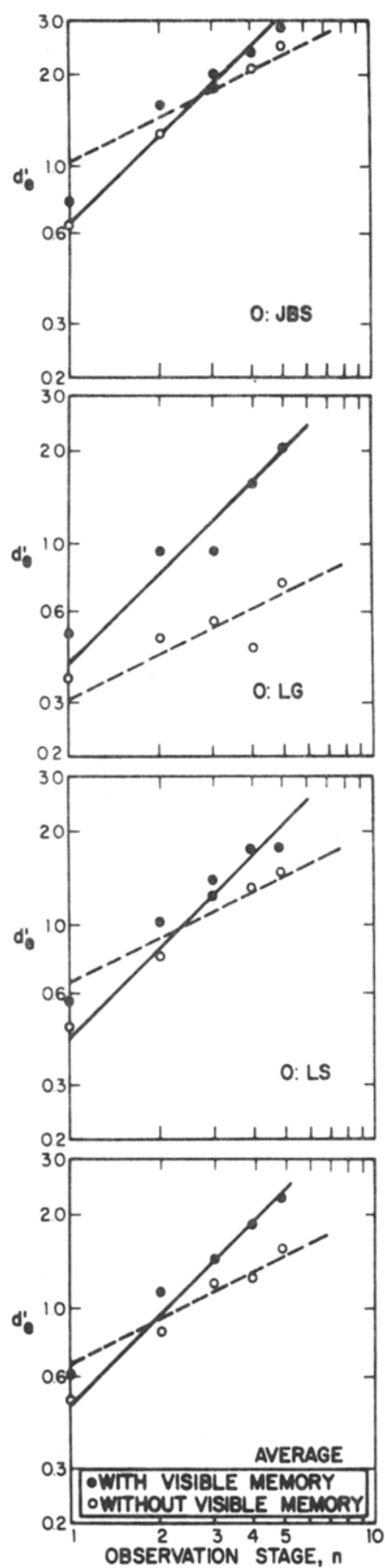

Figure 9. Experiment 1. The detection index, de, over the five observation stages for three observers, both with and without visible memory. Best-fitting (least-squares) lines with slopes of one and of one-half are shown as references for the with- and withoutvisible memory conditions, respectively. the first choice was incorrect, were $59 \%$ and $58 \%$, $32 \%$ and $30 \%$, and $43 \%$ and $35 \%$, for the two memory conditions and the three observers, respectively. These are all clearly greater than the chance percentage of approximately $14 \%$, and indicate that a substantial amount of information is conveyed by the second choice.

Predicting identification from detection. The model that predicts the correct detection-plusidentification ROC from the detection ROC is strongly supported by the data shown in Figure 11 (three observers, two memory conditions). In relatively few cases is there room for a line to connect predicted and obtained points. The average absolute discrepancies in percentage units for the three observers and two memory conditions (listing Condition a, visible memory, first) are 2.9 and 4.4, 4.0 and 5.3, and 3.1 and 3.8, respectively. Taking account of sign of deviation, the errors of prediction are -2.5 and $+1.4,-0.1$ and $+3.3,-0.3$ and -1.5 , where negative numbers indicate obtained values less than those predicted.

\section{Experiment 2}

Experiment 2 was conducted to determine if the data looked much the same, and if the model predicting identification from detection was as successful, when the signals were somewhat more complex. In this experiment, the signal was one or another of the five patterns of lines described earlier: a pattern consisted of a particular 3 of 16 possible lines. Just one "memory" condition was conducted, with each stimulus stage left visible throughout a trial. A larger number of trials was presented in this condition (320).

Detection ROCs. The detection ROCs in Figure 12 look familiar: reasonably straight lines with slopes tending to decrease as detectability increases (see Green \& Swets, 1966, 1974).

Detection accuracy over time. The index $\mathrm{d}_{\mathrm{e}}^{\prime}$ is seen in Figure 13 to increase approximately in proportion to the number of preceding observation stages.

Detection and identification accuracy over time. Performances on the two tasks become more accurate over time in related fashion; see Figure 14.

Accuracy of second choices. Again, second-choice identification responses are correct with greater-thanchance accuracy: $50 \%, 41 \%$, and $49 \%$ for the three observers, relative to the chance level of $25 \%$.

Predicting identification from detection. The prediction of detection-plus-identification from detection alone is less accurate here than in the previous experiment; see Figure 15. The average absolute discrepancies in percentage units between predicted and obtained points are 5.1, 15.9, and 5.9, for the three observers. These discrepancies are about 2 and 3 points greater than in Experiment 1 for the first (J.B.S.) and third (L.S.) observers, respectively, and 
(a) WITH VISIBLE MEMORY
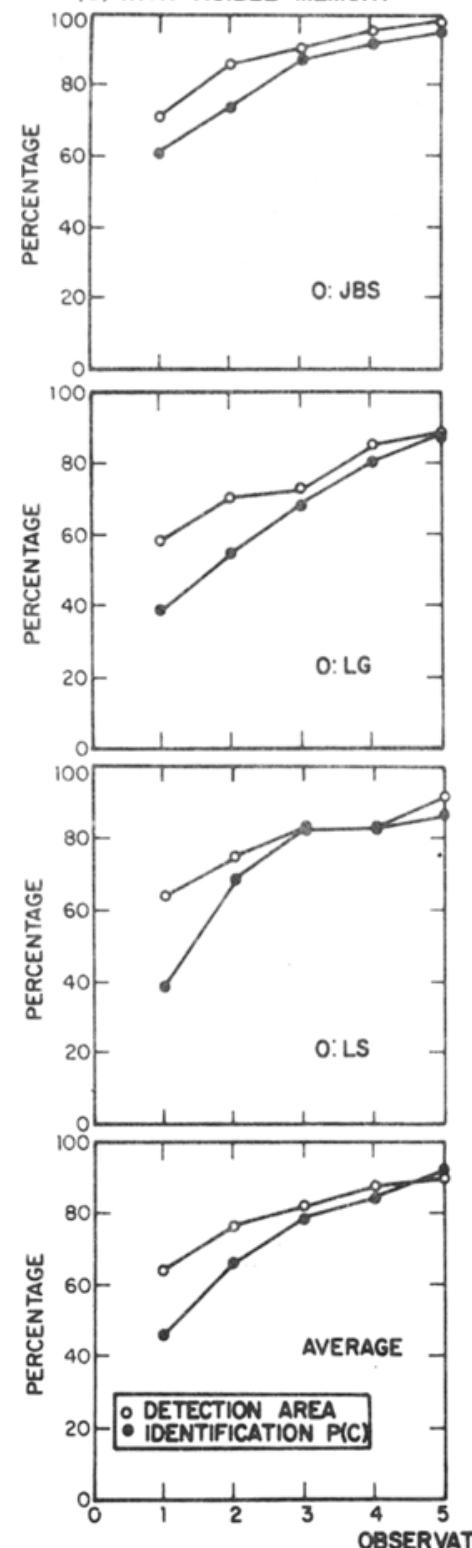

(b) WITHOUT VISIBLE MEMORY
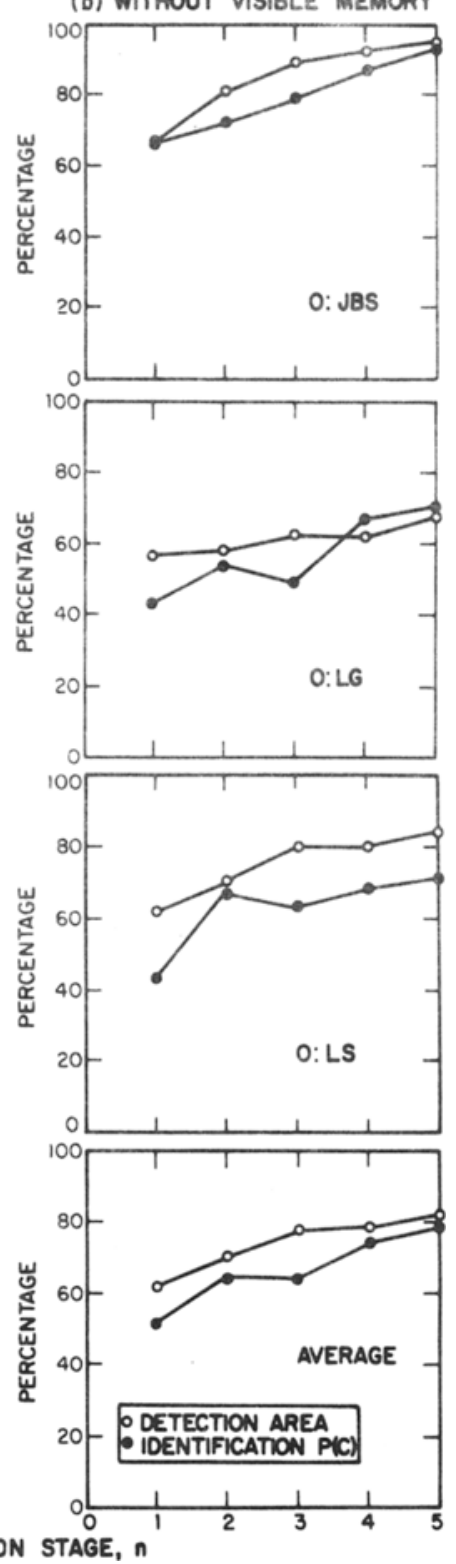

Figure 10. Experiment 1. The area under the ROC curve and the percentage of correct responses over observation stages for three subjects: (a) with and (b) without visible memory.

about 12 points greater for the second observer (L.G.). The average signed deviations are almost identical to the absolute deviations, with obtained values consistently greater than predicted. Why the obtained values are greater than the predicted values, which are supposed to be optimal relative to detection performance, remains to be determined. One possibility is that the forced identification response can be based on the detection of a single line, whereas the detection response might conservatively be based on the likelihoods of two or three lines. What is relatively a depressed detection performance would yield a depressed prediction of ideal identification performance. This possibility was suggested by the subjective report of one of the observers (J.B.S.).

\section{SUMMARY AND DISCUSSION}

The process envisioned in Nolte's model for detection and identification, and in Broadbent's analogy- 

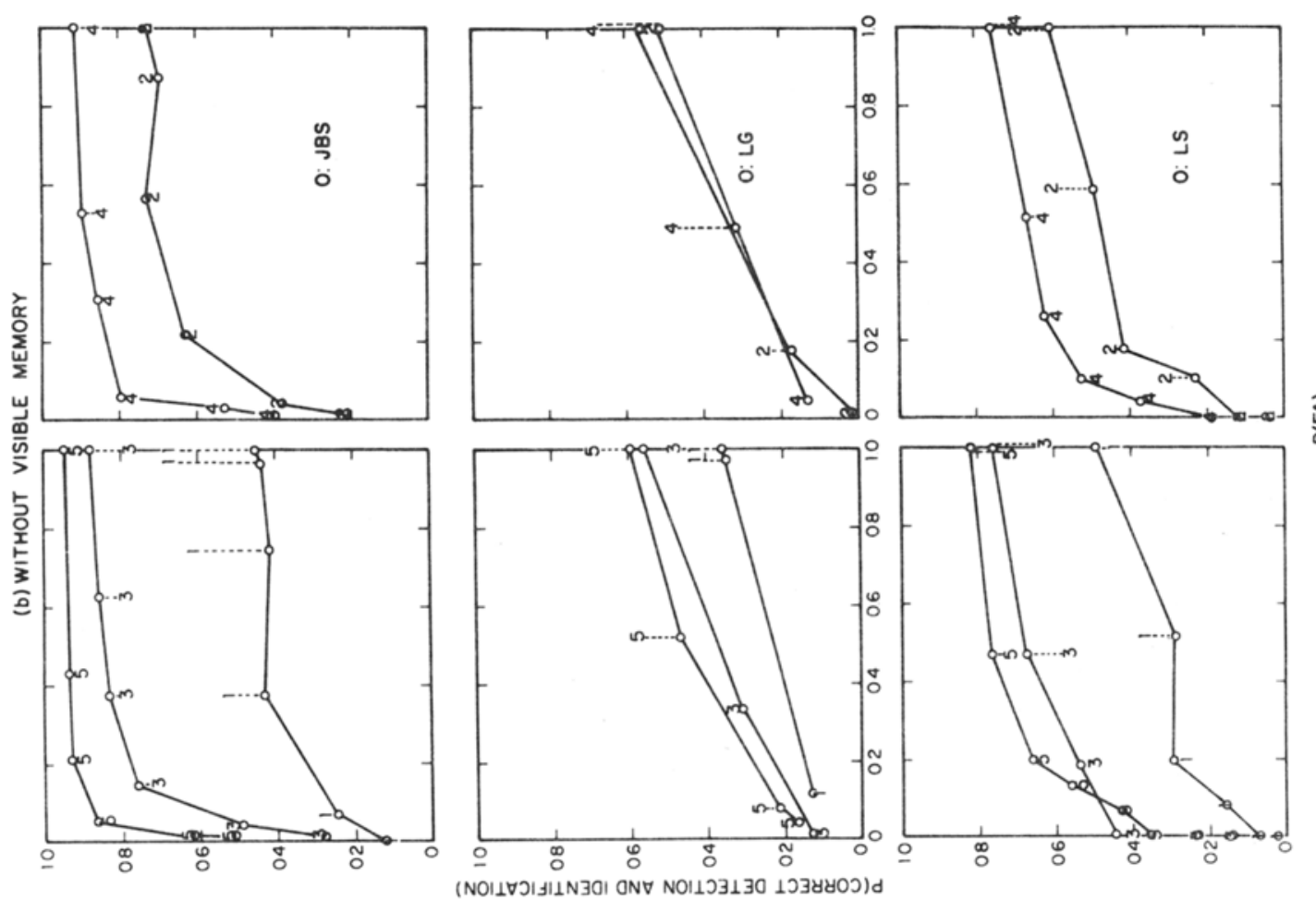

要
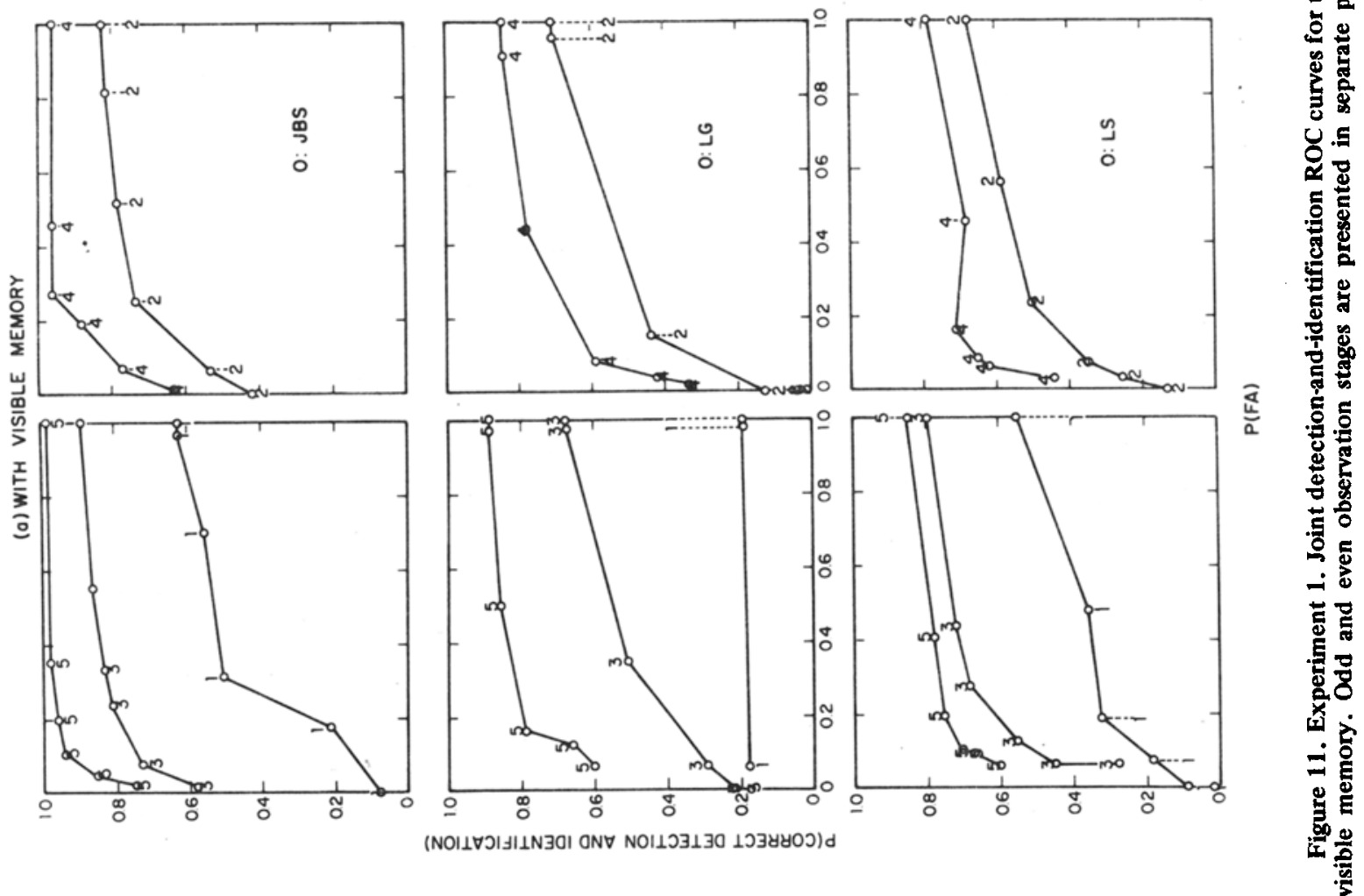

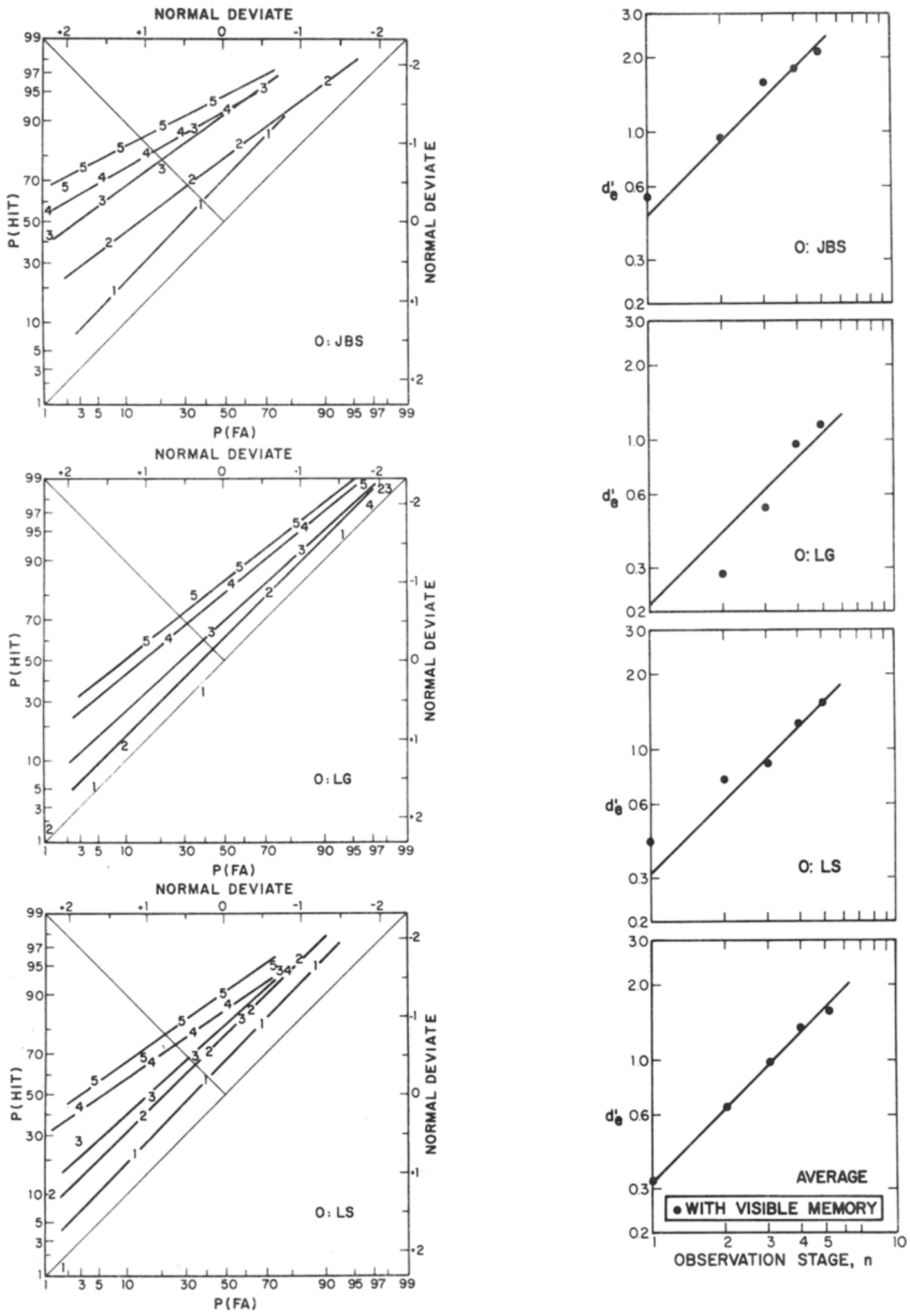

Figure 12. Experiment 2. Detection ROCs for each of the five stages of observation, for three observers.

Figure 13. Experiment 2. The detection index, $d_{e}^{\prime}$, over the five observation stages for three observers. A least-squares-fit line with slope of unity is shown as a reference. 

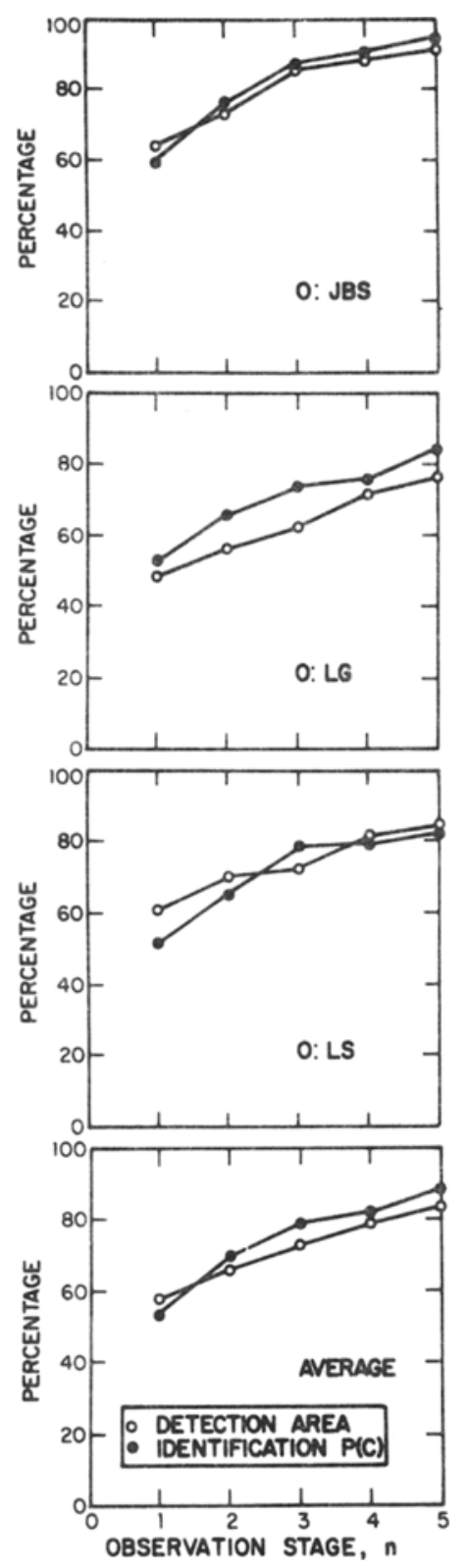

Figure 14. Experiment 2. The area under the ROC curve and the percentage of correct responses over observation stages, for three subjects.

wherein the observer updates probability estimates separately for each signal under consideration, and uses them for both kinds of response-is qualitatively consistent with our experimental results. Detection and identification indices grow over time together in a smooth fashion; second-choice identification probabilities exceed chance by a considerable margin, indicating access by the observer to current data on several signal possibilities. As we have said, we adduce Nolte's model and present our related experimental results because we suppose them to have heuristic, conceptual value. Clearly, more work is required to relate the fine structure of human behavior to the detection/identification process envisioned for the adaptive optimum receiver.

We observe, as an empirical aside, that the rate of growth of accuracy of both detection and identification is greater when all stimulus presentations in a trial remain available to the observer than when the observer must depend on memory for previous observations in a trial. That is to say, the assumptions made for the optimum receiver-that it extracts the relevant sensory information, and stores and updates the appropriate probabilities, with no loss occasioned by making piecemeal observations over time-are too strong for our human observers. Again, we defer further development of this point and the spelling out of its implications for method and practice.

Principal objectives of this paper have been to point to the relationship between identification and detection that Starr et al. have developed and reported in another context, and to report our attempt to extend this model's range of application. The model of Starr and his associates is seen to fit our data on simple location very well, that is, in Experiment 1, in which the task was to locate a line signal in one of eight bins or columns. The model also fits reasonably well the data of Experiment 2, in which the signal was one of five sets of three lines. The task of Experiment 2 can also be considered one of localization, of course, but the use there of simulated spectrographic patterns is suggestive of more general identification tasks.

To get some preliminary indication of the ability of the model to treat auditory signals, we have applied the model to Lindner's (1968) data on the detection and identification of two tones. The prediction was uniformly low, but deviated on the average (of four observers and four criteria) by only 3.0 percentage units. The discrepancies for the four observers individually were $2.1,4.6,3.8$, and 1.3.

One impact of these results is that time-consuming identification tests need not be conducted in perceptual situations to which the model applies-identification performance is predictable from detection performance. For this practical reason, as well as in the interest of theory, the limits of the model should be determined. We submit that the limits will have to be empirically determined. Though the main assumptions of the model-orthogonal and equal-energy signals-are clear and restrictive, we have other evidence that a model based on those assumptions can be rather robust. For example, Green and Birdsall (1964) have shown that the so-called one-of-Morthogonal-signals model (see Nolte \& Jaarsma, 1967) accounts well for the effect of vocabulary size in tests of speech perception. Moreover, a companion paper to this one (Swets et al., Note 2) presents evidence 

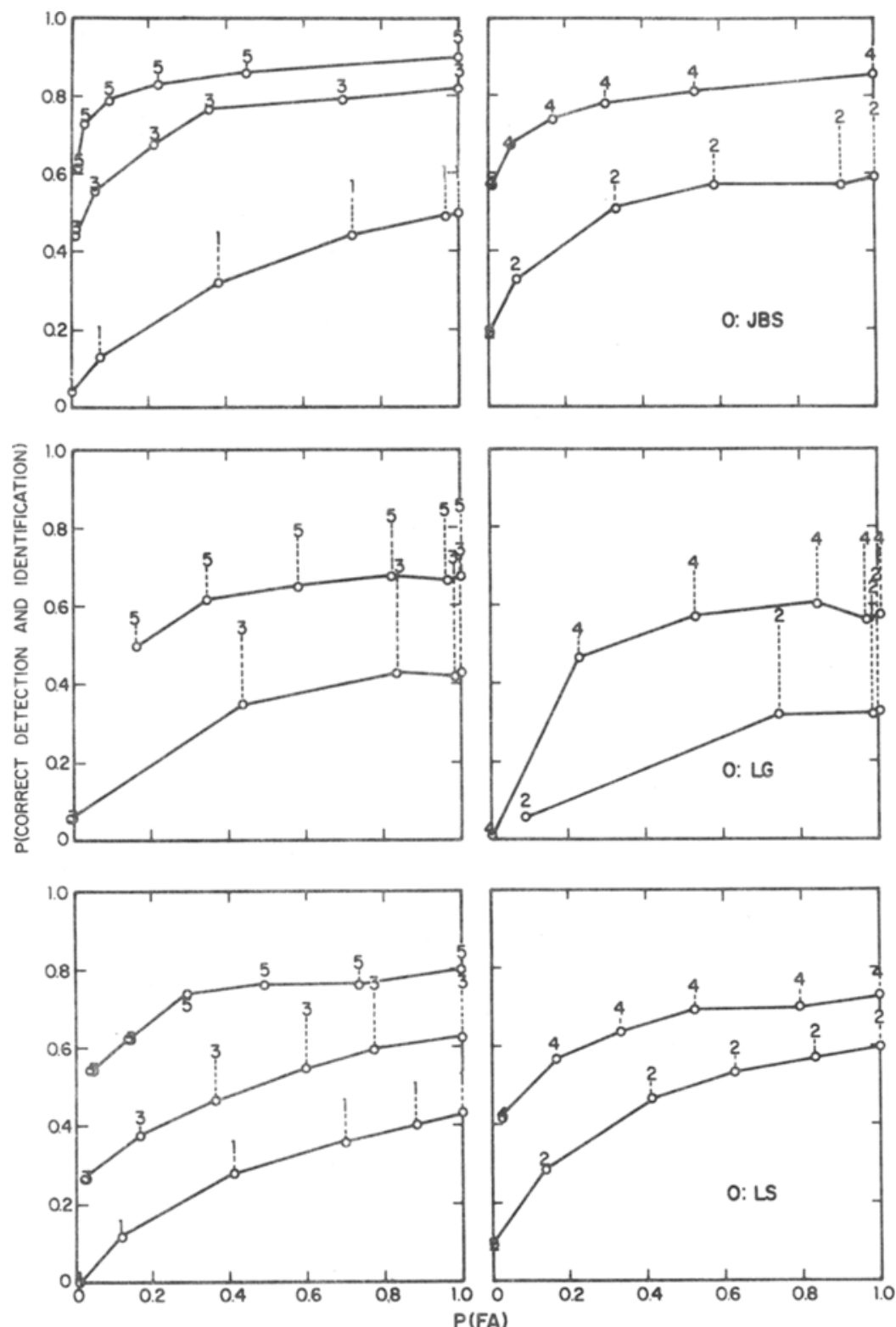

Figure 15. Experiment 2. Joint detection-and-identification ROC curves for three observers for each of five observation stages. Odd and even observation stages are presented in separate panels for clarity. The connected circles show values predicted by the model.

that the model predicts well the identification of real spectrographic patterns when the patterns to be identified differ according to their major psychological dimensions.

\section{REFERENCE NOTES}

1. Getty, D. J., Swets, J. A., Swets, J. B., \& Green, D. M. On the prediction of confision matrices from similarity judgments. Manuscript in preparation. 1978.
2. Swets, J. A., Green, D. M., Getty, D. J., \& Swets, J. B. Identification and scaling of complex visual patterns. Manuscript submitted for publication, 1978.

\section{REFERENCES}

Broadbent, D. E. Decision and stress. London and New York: Academic Press, 1971.

Green, D. M., \& Birdsall, T. G. The effect of vocabulaty on articulation score. In J. A. Swets (Ed.), Signal detection 
and recognition by human observers. New York: Wiley, 1964. Pp. 609-619.

Green, D. M., \& Swets, J. A. Signal detection theory and psychophysics. New York: Wiley, 1966. (Reprinted by Krieger, Huntington, New York, 1974.)

LINDNER, W. A. Recognition performance as a function of detection criterion in a simultaneous detection-recognition task. Journal of the Acoustical Society of America, 1968, 44, 204-211.

Nolte, L. W. Theory of signal detectability: Adaptive optimum receiver design. Journal of the Acoustical Society of America, 1967, 42, 773-777.

Nolte, L. W., \& JaArsma, D. More on the detection of one of $M$ orthogonal signals. Journal of the Acoustical Society of America, 1967, 41, 497-505.

Starr, S. J., Metz, C. E., Lusted, L. B., \& Goodenough, D.
J. Visual detection and localization of radiographic images. Radiology, 1975, 116, 533-538.

Swers, J. A., Birdsall, T. G. The human use of information, III. Decision-making in signal detection and recognition situations involving multiple alternatives. Transactions of the Institute of Radio Engineers, Professional Group on Information Theory, 1956, IT-2, 138-165.

Swets, J. A., \& Birdsall. T. G. Deferted decisions in human signal detection: A preliminary experiment. Perception \& Psychophysics, 1967, 2, 15-28.

Swets, J. A., \& Birdsall, T. G. Repeated observation of an uncertain signal. Perception \& Psychophysics, 1978, 23. 269-274.

(Received for publication September 19, 1977; revision accepted February 14, 1978.) 